\title{
Production et transformation du lait en région agropastorale au Sénégal : cas de la zone périurbaine de Kolda
}

\author{
M. Ba Diao ${ }^{1 *}$ C.D. Senghor ${ }^{2}$ \\ B. Diao ${ }^{3}$ E. Thys ${ }^{4}$
}

\section{Mots-clés}

Bovin - Lait - Commercialisation Traitement - Budget - Rentabilité Sénégal.

\begin{abstract}
Résumé
Au Sénégal, l'installation d'étables laitières privées autour des grands centres urbains a été encouragée depuis 1982. Ceci fut également le cas dans la région de Kolda. Dans le but d'analyser la filière, une étude a été réalisée de mai à septembre 2000, combinant la méthode de budget partiel au niveau de la production et un diagnostic participatif impliquant 114 intervenants pour analyser les différentes composantes de la filière. En dépit de plusieurs années de vulgarisation, seulement 3 p. 100 des vaches laitières ont été concernées par la stabulation partielle de saison sèche. L'analyse du budget partiel a pourtant montré que l'opération était rentable, laissant, après soustraction de la valeur de I'autoconsommation, un disponible de 17532 Fcfa ou de 8907 Fcfa par vache, selon que le producteur commercialisait le lait lui-même ou non. La majorité de la production est passée par le système traditionnel de transformation (lait caillé et huile de beurre). Les unités de transformation artisanales de la région, dont une fromagerie, n'auraient ainsi drainé que 13 p. 100 de la production de la saison sèche et 8 p. 100, de celle de la saison des pluies. La fraîcheur des produits pasteurisés de ces unités ne pouvait être garantie au-delà d'une semaine ce qui a limité I'accès aux grands marchés. A chaque échelon, les contraintes et les opportunités ont été analysées afin d'améliorer la dynamique de la filière lait.
\end{abstract}

\section{INTRODUCTION}

En Afrique sahélienne, le développement des villes, consécutif à l'accroissement démographique et à l'exode rural, rend de plus en plus difficile leur approvisionnement en produits alimentaires en général et en produits laitiers en particulier. Dans la plupart des pays, cette demande accrue entraîne une augmentation des importations. Cependant, cette solution devient de plus en plus difficile à mettre en œuvre compte tenu de la hausse des coûts d'importation (14).

\footnotetext{
1. Isra/Lnerv, BP 3120, Dakar, Sénégal

2. Performances Management Consulting, BP 22 352, Dakar Ponty, Sénégal

3. Sud-Vetagro, BP 139, Kolda, Sénégal

4. Institut de médecine tropicale Prince Léopold, département de Santé animale,

Nationalestraat 155, B- 2000 Antwerpen, Belgique

* Auteur pour la correspondance

Tél. : +2218323678; fax : +221832 2118 ; e-mail : mbadiao@sentoo.sn
}

Pour limiter la dépendance du Sénégal vis-à-vis de ces importations, l'Etat a encouragé, depuis 1982, l'installation d'étables laitières privées autour des grands centres urbains (1). Cette option, qui reposait sur l'importation et l'exploitation des races laitières exotiques, a vite montré ses limites (4). D'autres initiatives ont vu le jour au début des années 1990. Qu'ils s'agissent de l'organisation des circuits de collecte du lait (cas du projet Nestlé à Dahra), du programme d'insémination artificielle bovine (cas du Projet d'appui à l'élevage, Papel), tous ces programmes ne se sont intéressés qu'à un seul maillon de la filière. Or, l'enjeu des études sur le lait réside dans l'analyse de la diversité au sein de chaque maillon de la filière et de l'importance des interconnexions et des flux entre acteurs de la filière (15). Il est également connu qu'une filière aussi longue que peut être la filière laitière représente un réel support de développement des zones rurales, pour peu que soient réunies les conditions nécessaires à la production et à la transformation du lait $(6,7)$. L'analyse de filière constitue dès lors un outil puissant pour dresser un diagnostic des activités en cours (3). 
Dans la région de Kolda, les actions conjuguées de différents programmes de recherche et de développement réalisées, d'une part, par la Sodefitex ${ }^{1}$ et l'Isra-Crz ${ }^{2}$ (9), et, d'autre part, par le Primoca ${ }^{3}$ ont permis d'accroître la production laitière en saison sèche pour l'autoconsommation et la commercialisation autour des villes de cette région. L'augmentation des quantités de lait mises en marché y a favorisé l'émergence d'unités artisanales de transformation de lait $(9,15)$.

Le présent travail a eu pour objectif d'analyser les contraintes et opportunités de développement de cet embryon de filière structurée. L'accent a été mis sur les liens horizontaux et verticaux qui existaient entre les agents économiques, sur le degré de coordination entre les intervenants et sur les différentes interactions. En amont, ont été analysés les systèmes de production en présence et les gains attendus des actions d'amélioration. En aval, ont été caractérisés les modes de transformation et de commercialisation du lait par les éleveurs, et les unités artisanales pour clarifier leurs options respectives retenues.

\section{MATERIEL ET METHODES}

\section{Site d'investigation}

La zone d'étude a été circonscrite dans un rayon de 15 à $25 \mathrm{~km}$ autour des villes de Kolda, Sédhiou et Vélingara qui sont respectivement les chefs-lieux des départements de même nom. Ces trois départements forment la région de Kolda (figure 1) qui fait partie

${ }^{1}$ Société de développement des fibres textiles

${ }^{2}$ Centre de recherches zootechniques de l'Institut sénégalais de recherches agricoles

${ }^{3}$ Projet intégré pour la Moyenne Casamance de l'entité agroécologique de la Casamance, zone la plus arrosée du Sénégal (800 à $1200 \mathrm{~mm}$ par an). La région offre un paysage de plateaux drainés par un réseau hydrographique saisonnier, couvert par une forêt à feuilles caduques, des taillis de combrétacés et, par endroits, des peuplements de bambous. La strate herbacée contient un tapis dense de graminées vivaces.

Deuxième région pour l'élevage, Kolda compte 18 p. 100 du cheptel bovin du pays (10). L'élevage de la race taurine Ndama est intégré à l'agriculture dans un système de production agropastoral de type traditionnel. L'essentiel de l'alimentation provient des pâturages naturels, mais les pratiques culturales permettent d'offrir au bétail des sous-produits de récolte et de transformation agroindustrielle (fane d'arachide, tiges de mil et de maïs, graine de coton, tourteau de sésame). La prophylaxie sanitaire se limite à la campagne nationale de vaccination contre la péripneumonie contagieuse bovine. Sa population, dispersée en petits villages, est en majorité composée de Peuls sédentarisés de longue date.

\section{Méthodologie}

La méthode de diagnostic participatif (12) a été appliquée pour réaliser un état des lieux des différents maillons de la filière lait et pour effectuer une revue des contraintes socioéconomiques et technologiques ainsi que des opportunités de développement de la filière. Les informations ont été recueillies par une équipe pluridisciplinaire grâce à des enquêtes et entretiens participatifs réalisés de mai à septembre 2000. Les guides d'entretiens semi-directifs utilisés n'ont pas entravé la libre expression des interviewés, ils ont plutôt évité les digressions.

Au total, cent quatorze personnes ont été rencontrées, soit individuellement, soit en groupes. Il s'agissait de producteurs, transformateurs, vendeurs de lait, mais également de représentants des

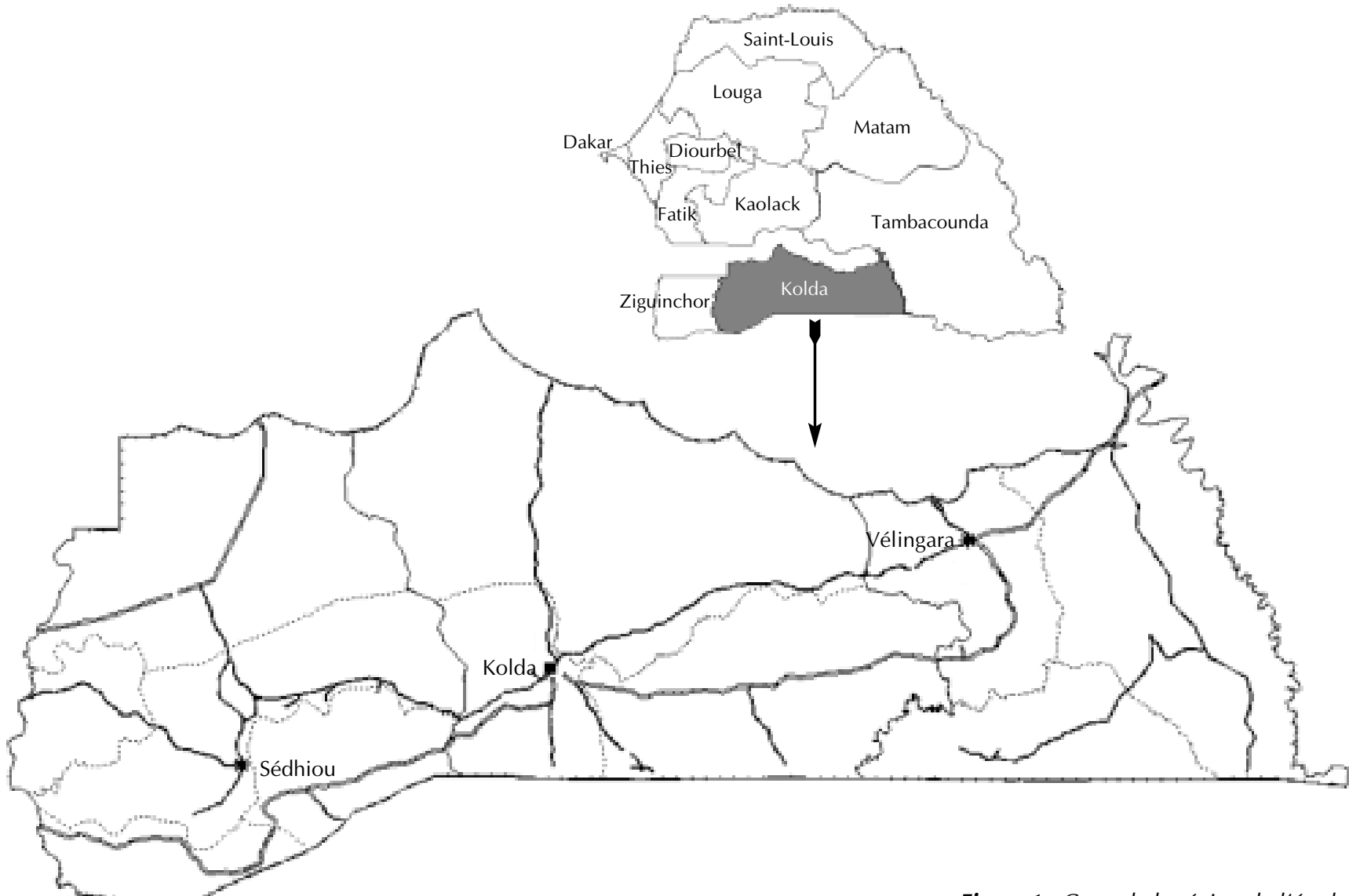

Figure 1 : Carte de la région de l'étude. 
structures d'encadrement, de recherche ou de crédit présentes dans la région. Par la méthode du budget partiel, la rentabilité des opérations de stabulation a été évaluée pour une durée de trois mois sur un échantillon de 15 producteurs, 12 autres producteurs ayant servi de témoins. Les six unités de transformation en activité dans la région ont été visitées. Leur mode d'organisation et de fonctionnement a été analysé, la saisonnalité de la quantité de lait réceptionnée évaluée. La rentabilité financière de l'unité de transformation qui possédait l'organisation la plus structurée a été étudiée. La structure des prix des produits laitiers a été étudiée tout le long de la filière.

\section{RESULTATS ET DISCUSSION}

La figure 2 donne une indication des différentes relations existant au sein de la filière des produits locaux dans la région de Kolda.

\section{Caractérisation des systèmes de production}

Le lait a été produit essentiellement par des troupeaux traditionnels de race Ndama. Le pâturage naturel a été la principale source alimentaire des animaux. Très peu de vaches (moins de 3 p. 100) ont été concernées par la technique de stabulation partielle en saison sèche. En moyenne, deux à trois couples veaux-vaches en production ont été complémentés par troupeau. La quantité de graines de coton distribuées après le pâturage a été en moyenne de $1,5 \mathrm{~kg}$ par vache par jour. Le tableau I montre que les vaches complémentées ont produit deux fois plus de lait que celles qui n'ont pas reçu de complément (1,57 1/jour contre 0,71 1/jour en moyenne pendant trois mois). Le budget partiel (tableau I) a mis en évidence la rentabilité de ce type d'opération. La complémentation a permis de disposer d'un revenu de 8907 Fcfa par vache, sans prendre en compte la valeur de l'autoconsommation qui a été de 18,6 p. 100.

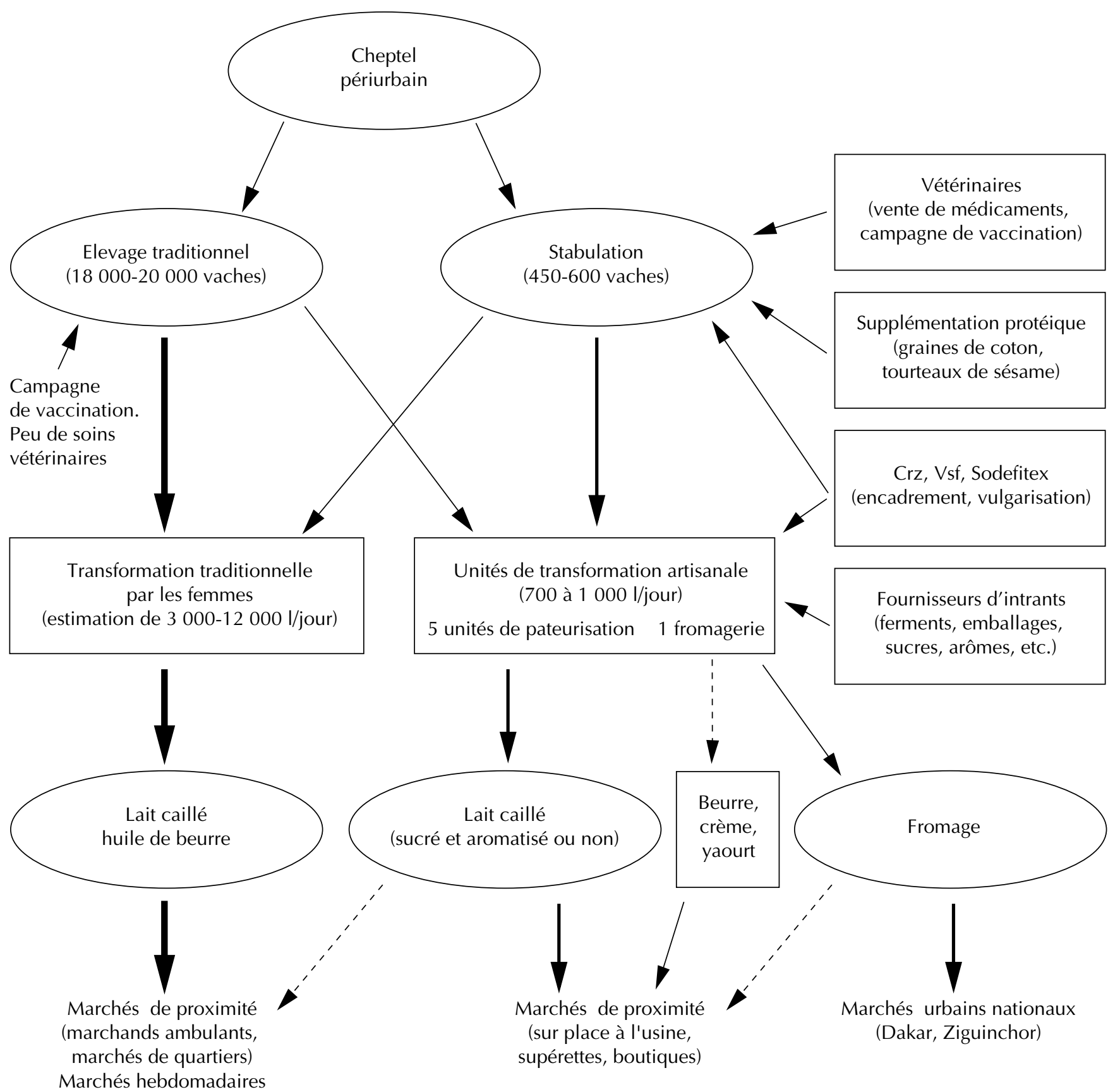

Figure 2 : Schéma global de la filière lait et interrelations entre acteurs dans les zones périurbaines de Kolda (Source : enquêtes 2000). 


\section{Tableau I}

Budget partiel des opérations (complémentation vs sans complémentation) en saison sèche

\begin{tabular}{|c|c|c|}
\hline \multirow[t]{2}{*}{ Rubrique } & \multicolumn{2}{|c|}{ Complémentation } \\
\hline & & sans \\
\hline Nombre de producteurs & 15 & 12 \\
\hline Vaches traites/producteur & 2,8 & 3,7 \\
\hline Production laitière totale par vache & 141,2 & 64,0 \\
\hline Autoconsommation (litres) & 26,2 & 28,0 \\
\hline Commercialisation (litres) & 115,0 & 36,0 \\
\hline Prix au producteur (Fcfa/l) & 200 & 200 \\
\hline \multicolumn{3}{|l|}{ Données par vache (Fcfa) } \\
\hline Produits & 28240 & 12800 \\
\hline Valeur autoconsommation (VA) & 5240 & 5600 \\
\hline Valeur commercialisation (VC) & 23000 & 7200 \\
\hline Coûts variables monétaires & 11272 & 1939 \\
\hline Alimentation & 10273 & 0 \\
\hline Produits vétérinaires & 470 & 160 \\
\hline Livreurs de lait & 529 & 529 \\
\hline Berger & 0 & 1250 \\
\hline $\begin{array}{l}\text { Coûts d'opportunité variables } \\
\text { (mobilisation de la main d'œuvre) }\end{array}$ & 2821 & 0 \\
\hline Charges variables totales & 14093 & 1939 \\
\hline Marge brute (MB) & 14147 & 10861 \\
\hline Dotation aux amortissements (DA) & 1570 & 0 \\
\hline Revenu net (RN) & 12577 & 10861 \\
\hline Revenu disponible (par vache) (RD) & 8907 & 5261 \\
\hline Prix de revient par litre & 117,6 & 30,3 \\
\hline Revenu disponible par litre de lait produit & 63 & 82 \\
\hline Revenu disponible par producteur (Fcfa) & 25060 & 19480 \\
\hline
\end{tabular}

Source : données d'enquêtes (2000)

$\mathrm{NB}: \mathrm{RN}=\mathrm{MB}-\mathrm{DA} ; \mathrm{RD}=\mathrm{MB}-\mathrm{VA}$

Ces gains financiers par vache ont pu être doublés (17 532 Fcfa) chez des producteurs qui ont vendu directement leur lait sur le marché à 275 Fcfa le litre.

A ces résultats financiers, il fallait ajouter la production de fumier destiné à l'amélioration de la fertilité des sols, les gains de poids observés chez les vaches $(120 \mathrm{~g} / \mathrm{j})$ et les veaux $(110 \mathrm{~g} / \mathrm{j})$ et la réduction de l'intervalle entre vêlages $(5,8)$. La stabulation a donc eu des objectifs multiples : assurer une production de lait et de fumier de qualité tout en préservant la fonction de reproduction (9).

Toutefois, ces résultats auraient pu être améliorés au regard des observations de terrain. Les étables visitées ont été conçues pour la production de fumure organique et la stabulation d'animaux de trait, mais les abreuvoirs n'ont pas été prévus, alors que la production laitière exigeait un accès permanent à l'eau. De même, la période optimale pour la stabulation laitière n'a pas été respectée, les intrants n'ayant pas été disponibles sur le marché au moment le plus favorable pour le démarrage de la stabulation (janvierfévrier). Les producteurs ont également été confrontés au problème d'accès aux sous-produits, n'ayant pas eu les moyens de financer eux-mêmes un stock suffisant de graines de coton. L'apport en graines de coton recommandé de deux kilogrammes par vache et par jour (8) n'a pas été suivi, d'où des résultats très variables selon les exploitations (production par vache d'un à trois litres, voire moins d'un litre et tarissement de vaches assez fréquent). Le faible nombre de vaches en stabulation a augmenté le coût de la main d'œuvre (2 821 Fcfa par vache). Ce coût pourrait être réduit avec l'augmentation du nombre de femelles complémentées (9).

Plusieurs raisons ont expliqué la faible pratique de la stabulation, en dépit de plusieurs années de vulgarisation de cette technique. L'absence de produits financiers adaptés permettant aux éleveurs de financer leurs investissements (constructions d'étables, achat et constitution éventuels de stocks d'intrants de supplémentation, etc.), la faible tradition entrepreneuriale chez les éleveurs et le caractère encore incertain des gains attendus de la production de lait par la pratique de la stabulation ont été autant de facteurs qui ont expliqué la faible appropriation de cette technique. Par ailleurs, la faiblesse des liens avec les unités de transformation, qui ne pouvaient, en l'absence de maitrise des marchés, se risquer à formaliser avec les producteurs de lait des contrats leur assurant une sécurité d'écoulement de leur production, a été un facteur de taille.

La production laitière a donc été pratiquée dans le cadre d'une économie tournée vers l'autoconsommation et les échanges locaux (13). Le lait s'est bien prêté à cette double fonction d'alimentation quotidienne de la famille et de vente en de petites quantités au marché hebdomadaire ou en ville. Il a été mis en marché par de petits producteurs qui ont présenté ainsi une offre atomisée, soit directement aux consommateurs, soit par des intermédiaires.

\section{Organisation de la collecte et de la transformation}

Une grande partie de la production locale périurbaine (77 p. 100 en saison sèche et 92 p. 100 en saison des pluies) serait passée par le système traditionnel de transformation. Les acteurs étaient des femmes d'éleveurs et des femmes transformatrices résidant en ville. Les produits finis ont été le lait caillé et l'huile de beurre.

Les unités de transformation n'ont drainé qu'une faible partie du lait local mis en marché. Le tableau II montre que leur capacité a été assez faible, seules deux d'entre elles ont dépassé les cent litres de lait par jour. Les techniques de transformation utilisées ont été artisanales avec, cependant, un souci de respect des règles minimales d'hygiène (sensibilisation des éleveurs sur l'hygiène à la traite, contrôle du lait à la livraison à l'unité, nettoyage et désinfection des récipients de collecte du lait). Les produits proposés par ces unités, excepté la fromagerie de Sédhiou, ont été limités au lait caillé et à l'huile de beurre produite à partir de crème de lait prélevé (1,1 p. 100 par litre), après le barattage manuel et la cuisson. Le lait caillé a été proposé nature ou aromatisé, sucré ou non, en sachets d'un quart de litre ou d'un demi-litre.

La fromagerie Ajapds à Sédhiou est apparue comme l'unité la plus importante et la plus moderne. Une gamme assez large de fromages y était produite (12 types), mais deux produits ont fait plus de 70 p. 100 des ventes : la mozzarella, en général utilisée pour la fabrication de pizzas, et l'italico, fromage ressemblant à l'emmental.

Un facteur important de la production laitière a été son caractère saisonnier très prononcé, nettement lié aux caractéristiques climatiques. Ceci a permis de distinguer deux périodes institutionnalisées par la fromagerie en accord avec les producteurs :

- une période de haute lactation correspondant à la saison des pluies, caractérisée par une abondance fourragère qui dure de juillet à novembre. Au cours de cette période, où les excédents de production ont été fréquents, le litre de lait livré a été payé $150 \mathrm{Fcfa}$. C'était une période favorable à la fromagerie qui a pu atteindre le maximum de sa capacité de production $(500 \mathrm{l} / \mathrm{j})$, 
Tableau II

Caractéristiques des différentes unités de transformation

\begin{tabular}{|c|c|c|c|c|c|c|}
\hline \multirow[t]{2}{*}{ Localité } & \multirow[t]{2}{*}{$\begin{array}{l}\text { Nom de l'entreprise } \\
\text { (année de création) }\end{array}$} & \multicolumn{2}{|c|}{$\begin{array}{l}\text { Niveau de collecte } \\
\text { (litres/jour) }\end{array}$} & \multirow[t]{2}{*}{$\begin{array}{l}\text { Organisme } \\
\text { d'appui }\end{array}$} & \multirow[t]{2}{*}{$\begin{array}{l}\text { Produit } \\
\text { transformé }\end{array}$} & \multirow[t]{2}{*}{ Marché } \\
\hline & & Saison sèche & Saison des pluies & & & \\
\hline \multirow[t]{4}{*}{ Kolda } & Le berger (1996) & 80 & $50-60$ & Ong Vsf/Sodefitex & $\begin{array}{l}\text { Lait caillé sucré, } \\
\text { non sucré }\end{array}$ & $\begin{array}{l}\text { Marché } \\
\text { urbain de } \\
\text { Kolda }\end{array}$ \\
\hline & Le fermier (1998) & 200 & 150 & Néant & $\begin{array}{l}\text { Lait caillé sucré } \\
\text { aromatisé, } \\
\text { huile de beurre }\end{array}$ & $\begin{array}{l}\text { Marchés de } \\
\text { Kolda, Diaobé, } \\
\text { Ziguinchor, }\end{array}$ \\
\hline & Teesitoo (1998) & 80 & $50-60$ & Sodefitex & $\begin{array}{l}\text { Lait caillé sucré } \\
\text { et non sucré }\end{array}$ & $\begin{array}{l}\text { Marché de } \\
\text { Kolda }\end{array}$ \\
\hline & Kaggu (1998) & \multicolumn{3}{|c|}{ Arrêt en 2000, mais reprise des activités en fin 2002} & & \\
\hline Sédhiou & Fromagerie Ajapds (1996) & $100-250$ & $450-500$ & Primoca & $\begin{array}{l}\text { Fromage (beurre, } \\
\text { lait caillé, yaourt) }\end{array}$ & $\begin{array}{l}\text { Marchés de } \\
\text { Dakar, Sédhiou, } \\
\text { Ziguinchor }\end{array}$ \\
\hline \multirow[t]{2}{*}{ Vélingara } & Pinnal Fouladou (1997) & 60 & 50 & Fongs & $\begin{array}{l}\text { Lait caillé sucré } \\
\text { et non sucré }\end{array}$ & $\begin{array}{l}\text { Marché de } \\
\text { Vélingara }\end{array}$ \\
\hline & Unité Bouba Sow (1999) & 70 & $50-60$ & Néant & $\begin{array}{l}\text { Lait caillé sucré } \\
\text { et non sucré }\end{array}$ & $\begin{array}{l}\text { Marchés de } \\
\text { Vélingara, } \\
\text { Diaobé }\end{array}$ \\
\hline
\end{tabular}

Source : données d'enquêtes (2000)

NB : Diaobé est un marché hebdomadaire sous-régional qui polarise outre le Sénégal, la Gambie, la Guinée et la Guinée Bissau

au contraire des autres unités de pasteurisation qui ont été fortement concurrencées par la transformation traditionnelle parce qu'elles ont produit la même denrée, le lait caillé (5) ;

- une période de basse lactation en saison sèche, où le prix du litre est monté à $175 \mathrm{Fcfa}$ au producteur. C'était une saison difficile pour la fromagerie qui a eu du mal à atteindre les cent litres par jour, surtout à partir du mois de mars. Par ailleurs, les prix offerts par les consommateurs directs (250 à 300 Fcfa le litre) ont induit un afflux de lait vers le circuit informel de commercialisation qui a contribué à amplifier la chute des quantités collectées par la fromagerie.

Le tableau III donne des indications sur les résultats économiques de cette entreprise en 1999. L'unité a acheté 445001 de lait à un prix moyen de 157,3 Fcfa le litre livré à l'usine. La marge brute obtenue a montré que les charges financières ont été à peine couvertes. Le bilan aurait été largement négatif s'il avait été tenu compte de l'amortissement du matériel de transformation (offert gracieusement au groupement de producteurs) et du véhicule (prêté au groupement). L'analyse des dépenses a montré qu'il y aurait eu pléthore de personnel (26 p. 100 des charges totales). Dix personnes ont eu en charge la transformation et la commercialisation de 1601 en moyenne par jour de collecte. Des économies auraient pu être faites à ce niveau comme dans d'autres postes de dépense, par exemple celui du carburant pour le transport. Des analyses économiques sur une période de 10 années (15) ont montré que la rentabilité financière totale de ce genre d'unité ne pouvait être atteinte qu'à partir d'une transformation d'au moins $500 \mathrm{l}$ par jour (pour 300 jours ouvrables). Le niveau actuel de transformation a donc été faible et, s'il restait en l'état, il ne permettrait pas à l'avenir à la fromagerie de se maintenir et de renouveler son matériel de production.

\section{Commercialisation}

Dans le circuit traditionnel, le lait caillé était vendu en ville directement au consommateur ou par un intermédiaire, à l'aide d'une calebasse en forme de louche ou de petit pot en plastique dont la contenance a été très variable (de 50 à $125 \mathrm{ml}$ ). Les femmes d'éleveurs faisaient le porte-à-porte ou avaient des emplacements fixes dans les marchés. Les transformatrices de la ville vendaient à domicile. Le tableau IV montre que, dans l'ensemble, les prix de Kolda ont été supérieurs à ceux de Sédhiou. Dans le système de vente directe, les bénéfices ont pu être importants, aussi bien en lait caillé qu'en huile de beurre. En revanche, la vente à des revendeurs ou aux usines de transformation a été moins rentable. Dans la ville de Kolda, les marges ont pu aller de 48 à 223 Fcfa par litre de lait. Une première analyse a montré qu'au litre de lait c'était l'éleveur qui faisait le plus grand bénéfice, surtout si la vente était directe, sans intermédiaire. Cependant, cette position était très fragile, notamment à cause du coût des intrants alimentaires ( $50 \mathrm{Fcfa} / \mathrm{kg}$ de graines de coton en $1997,75 \mathrm{Fcfa} / \mathrm{kg}$ en 2000 et $120 \mathrm{Fcfa} / \mathrm{kg}$ en 2003).

Dans ce système, s'est également posé le problème de la qualité des produits, des conditions d'hygiène au niveau de la transformation et de la vente. L'huile de beurre s'est conservée en revanche plus longtemps (des semaines, voire des mois) et a emprunté généralement un circuit de commercialisation plus long. Les centres urbains éloignés, notamment Dakar, ont été la destination finale. La rareté du produit et les possibilités de conservation ont expliqué son prix élevé dans la zone de production.

Au niveau des unités de transformation, la pasteurisation a permis de mettre sur le marché domestique des produits de qualité 


\section{Tableau III}

Analyse économique d'une fromagerie artisanale - exercice 1999

\section{Tableau IV}

Formation des prix dans la filière lait à Kolda en saison sèche (2000)

Lcfa/l de lait ou
Lcfa/kg de fromage

\section{Tableau V}

Prix de quelques produits laitiers dans la ville de Kolda (2000)

conditionnement (ensachage). La commercialisation s'est faite par des glacières pour garder le produit à l'abri de la chaleur. Cependant, le caractère encore artisanal des unités et leurs équipements de production sommaires n'ont pas donné une qualité suffisante pour commercialiser les produits dans des circuits longs et distants, lesquels exigeaient une garantie de conservation d'au moins 21 jours (15). Les unités existantes n'ont pu garantir leurs produits au-delà d'une semaine de conservation sous froid et la standardisation du produit (acidité, texture) n'a pas été assurée. Le commerce des produits laitiers transformés au niveau des unités artisanales a donc été celui de proximité. Les unités installées ont visé essentiellement les marchés domestiques urbains (Sédhiou, Kolda, Vélingara et, épisodiquement, Diaobé). Elles ont cherché à satisfaire une demande locale non encore saturée, sans effort apparent de commercialisation dont les frais étaient, par ailleurs, réduits. La faiblesse des quantités produites ne les a guère obligées à entreprendre des efforts pour pénétrer des marchés plus lointains impliquant des frais de transport.

Cependant, l'implantation de ces produits aurait pu être durable en milieu urbain si on en avait amélioré la qualité car, au niveau des prix, la comparaison avec les produits à base de lait en poudre venant de Dakar a été largement en faveur des produits locaux (tableau V). De même, les efforts de respect de protocole de 
Cette dynamique de qualité a rendu les transformateurs plus exigeants pour la qualité du lait collecté et a favorisé une prise de conscience sur la nécessité de l'investissement et de l'amélioration des processus de transformation. Pratiquement tous les transformateurs ont été porteurs de projets d'augmentation de capacité et de modernisation, pour tenter de satisfaire des marchés plus larges avec la demande qui proviendrait des régions limitrophes : Guinée Conakry, Ziguinchor, Tambacounda et Gambie (15).

\section{- CONCLUSION}

Les unités de transformation du lait ont été des maillons essentiels de la filière laitière car ils ont mis à la portée des éleveurs la possibilité d'écouler leur lait. Ces unités ont induit de nouveaux comportements et de nouvelles pratiques chez les éleveurs, et de nouvelles exigences chez les consommateurs. Toutefois, elles ont toutes été de création récente et n'ont pas eu encore la taille critique pour garantir un niveau de production permettant une rentabilité financière et une présence permanente sur les marchés. Un changement d'échelle et de type de marché exigerait des compétences accrues pour la technologie de transformation, le management de l'unité et la gestion des problèmes, jusqu'ici inconnus des petits opérateurs : gestion du personnel, ordonnancement de la production et des livraisons, organisation de la distribution et commercialisation. Les innovations technologiques par des investissements seront nécessaires et permettront de garantir une grande qualité des produits, tout en restant le plus sobre possible dans le choix des équipements. L'alternative est donc soit l'adaptation des unités au nouveau contexte concurrentiel, soit de rester petit et de n'avoir comme perspectives que les marchés urbains de proximité de la région de Kolda.

Cependant, cette transformation souhaitée à l'aval de la filière exige également une amélioration des systèmes de production. Les performances de production laitière se caractérisent par leur forte saisonnalité. Ces tendances impliquent une fragilité du système de production laitière en zone pluviale, puisqu'elle dépend du climat. De plus, les conditions techniques et économiques sous-jacentes à la production laitière (faiblesse des disponibilités fourragères, difficulté d'acquisition des intrants alimentaires, insuffisance du conseil zootechnique, déficit du crédit, faiblesse de la capacité institutionnelle des producteurs) sont autant de contraintes qu'il importe de résoudre. Sinon, les espoirs de génération de revenus sur lesquels reposent les investissements dans les techniques de stabulation et la transformation du lait seraient compromis.

\section{Remerciements}

Les auteurs remercient l'ensemble des producteurs de la région de Kolda, les représentants des organisations paysannes, les responsables des projets de développement et des systèmes décentralisés de crédit pour leur disponibilité.

\section{BIBLIOGRAPHIE}

1. BA DIAO M., 1996. La production laitière au Sénégal : contraintes et perspectives. In : $3^{\text {e }}$ journées scientifiques du Réseau biotechnologies animales de l'Aupelf-Uref sur "Reproduction et production laitière », Rabat, Maroc, 26-28 septembre 1995. Tunis, Tunisie, Serviced, p. 63-73.

2. BALDE O., 1999. Etude de marché du lait pasteurisé à Kolda et Vélingara. Rapport de stage. Promer - cellule régionale de Kolda. Fez, Maroc, Sup'Management, 20 p.

3. BENCHARIF A., CHAULET C., CHEHAT F., KACI M., SAHLI Z., 1996. La filière blé en Algérie : le blé, la semoule et le pain. Paris, France, Editions Karthala, 238 p.

4. BROUTIN C., DIOKHANE O., 2000. La filière "lait et produits laitiers » au Sénégal. Dakar, Sénégal, Etude Gret/Tpa, 38 p.

5. COLLEUILLE A., DIEYE P.N., MBODJ A., 1999. La filière laitière en Haute Casamance et au Sénégal oriental. In : Actes de I'atelier de réflexion, Kolda, 8-9 avril 1999. Kolda, Sénégal, Isra-Crz / Sodefitex / Vétérinaires sans frontières, $15 \mathrm{p}$.

6. DE BOER J., 1981. Socio economic aspects of dairying in developing countries. J. Dairy Sci., 64: 2453-2462.

7. DE JONG R., 1996. Dairy stock development and milk production with smallholders. Doct. Thesis, Wageningen Institute of Animal Sciences, The Netherlands, $216 \mathrm{p}$.

8. DIEYE P.N., 1999. La filière laitière : quelques résultats de recherches en Haute Casamance. In : Actes de I'atelier de réflexion, Kolda, 8-9 avril 1999. Kolda, Sénégal, Isra-Crz / Sodefitex / Vétérinaires sans frontières, $4 \mathrm{p}$.
9. DIEYE P.N., FAYE A., SEYDI M., CISSE S.A., 2002. Production laitière périurbaine et amélioration des revenus des petits producteurs en milieu rural au Sénégal. Cah. Agric., $11: 251-257$.

10. DIREL, 1998. Les statistiques de l'élevage. Année 1997. Dakar Sénégal, direction de l'Elevage, ministère de l'Agriculture, 67 p.

11. DUTEURTRE G, 2000. L'organisation de la filière laitière autou de Niono. Montpellier, France, Cirad-emvt, 77 p. (Rapport de mission $\left.\mathrm{n}^{\circ} 10\right)$

12. ELLSWORTH L., DIAME F., DIOP S., THIEBA D., 1992. Le diagnostic participatif (Participatory Rural Appraisal). Dakar-Fann, Sénégal, Praap, $238 \mathrm{p}$.

13. GRIFFON M., 2000. Efficacité du marché et organisation dans I'agriculture et I'agroalimentaire en Afrique. Rapport de synthèse. Montpellier, France, Cirad-amis, $77 p$.

14. METZGER R., CENTRES J.M., THOMAS L., LAMBERT J.C., 1995 L'approvisionnement des villes africaines en lait et produits laitiers. Un potentiel pour un développement rural. Production et santé animales, vol. 124. Rome, Italie, FAO, 102 p.

15. Tdc-Sénégal, 2000. Les opportunités d'affaires dans la filière laitière de la région de Kolda. Dakar, Sénégal, Technologie développement consultance / Dynaentreprises / projet Usaid, 95 p.

Reçu le 07.01.2003, accepté le 19.09.2003 


\section{Summary}

Ba Diao M., Senghor C.D., Diao B., Thys E. Milk Production and Processing in the Agropastoral Region of Senegal: Case of Kolda Suburban Area

Setting up private dairy cowsheds on the outskirts of large urban centers has been encouraged in Senegal since 1982. This was thus the case for Kolda region. A study was carried out from May to September 2000 to analyze dairy production. To analyze the various production stages the study combined the method of partial budget at the production level and a participatory diagnosis with 114 participants. Only 3\% of dairy cows were partially housed during the dry season in spite of several years of popularizing work. The partial budget analysis did show, nevertheless, that the operation was profitable. After deducting the part of autoconsumption, it generated an income of 17,532 FCFA or 8907 FCFA per cow, depending on whether the producers sold the milk themselves or not. Most of the milk production was transformed traditionally (curds and butter oil). The craft transformation units in the area, including a cheese factory, absorbed only $13 \%$ of the production in the dry season and $8 \%$ in the rainy season. Access to large markets was limited by the fact that the storage life of products pasteurized by these units could not be guaranteed beyond one week. Constraints and opportunities were analyzed at every level with the aim of improving dynamics of dairy production.

Key words: Cattle - Milk - Marketing - Processing - Budget Profitability - Senegal.

\section{Resumen}

Ba Diao M., Senghor C.D., Diao B., Thys E. Producción y transformación de la leche en una región agropastoril de Senegal: caso de la zona peri urbana de Kolda

En Senegal, se ha impulsado, desde 1982, la instalación de establos de leche privados, alrededor de los grandes centros urbanos. Este es el caso también en la región de Kolda. Con el fin de analizar esta filial, se realizó un estudio, de mayo a septiembre 2000, combinando el método de presupuesto parcial en el ámbito de la producción y un diagnóstico participativo, implicando 114 participantes, para analizar los diferentes componentes de la filial. A pesar de varios años de divulgación, solamente $3 \%$ de las vacas de leche fueron estabuladas parcialmente durante la estación seca. El análisis del presupuesto parcial mostró, sin embargo, que la operación fue rentable, dejando, después de la substracción del valor del auto consumo, un monto disponible de 17532 Fcfa o 8907 Fcfa por vaca, dependiendo de si el productor comercializó la leche el mismo o no. La mayoría de la producción pasó por el sistema tradicional de transformación (leche cortada y aceite de mantequilla). Así, las unidades de transformación artesanales de la región, incluyendo una quesería, no produjeron más que $13 \%$ de la producción de la estación seca y $8 \%$ de la de las Iluvias. La frescura de los productos pasteurizados de estas unidades no pudo ser garantizada más de una semana, lo que limitó el acceso a los mercados grandes. En cada etapa, se analizan los obstáculos y las oportunidades, con el fin de mejorar la dinámica de la filial lechera.

Palabras clave: Ganado bovino - Leche - Mercadeo - Procesamiento - Presupuesto - Rentabilidad - Senegal. 